\section{Apparent Male Fertility in Musa Germplasm}

\author{
Boniface B. Dumpe ${ }^{1}$ and Rodomiro Ortiz ${ }^{2}$ \\ Plantain and Banana Improvement Program of the International Institute of \\ Tropical Agriculture, High Rainfall Station, PMB 008, Nchia Eleme, Rivers \\ State, Nigeria
}

Additional index words. banana, breeding, plantain, pollen stainability

\begin{abstract}
Current efforts to produce improved genotypes of plantain and banana (Musa spp.) depend on crossing female-fertile clones with accessions that produce viable pollen. Musa accessions (168) were screened for production of viable pollen based on staining with acetocarmine glycerol jelly. Diploid hybrids and landraces produced significantly more pollen than triploids and tetraploids, suggesting more successful crosses when using diploid accessions as male parents. There was a positive correlation between the amount of pollen produced and the level of viability in both hybrids $(r=0.65, P \leq 0.01)$ and landraces $(r=0.61, P \leq 0.01)$. This finding suggests that closely associated genetic factors determine these characteristics in Musa, while environmental conditions also may influence the quality and quantity of pollen produced. Pollen production at anthesis was absent in 28 accessions. Of the 140 accessions with pollen, 67 were sufficiently fertile for use as male parents in the breeding program.
\end{abstract}

Plantain and banana are important food crops and sources of revenue for small-holder farmers in the humid forest and mid-altitude agroecological regions of sub-saharan Africa (Vuylsteke et al., 1993a). About 70 million people in the region obtain more than $25 \%$ of their carbohydrates intake from these crops, and their gross value of annual production ranks above several other major food crops.

Most of the cultivated Musa, especially plantains, are threatened by black sigatoka, a destructive fungal leaf spot disease caused by Mycosphaerella fijiensis Morelet. This disease causes yield losses of $30 \%$ to $50 \%$ (Mobambo et al., 1993; Stover, 1983). Fungicidal control is expensive and poses health and environmental hazards. Breeding of improved cultivars with durable host plant resistance is generally considered the most appropriate control strategy (Vuylsteke et al., 1993b). The Plantain and Banana Improvement Program (PBIP) of the International Institute of Tropical Agriculture (IITA) relies on the fertilization of female-fertile partners by pollen of male parents to generate new hybrid genotypes with improved agronomic performance, including resistance to the black sigatoka disease (PBIP, 1993).

The rate of genetic improvement in cultivated Musa depends on the reproductive fertility (Vuylsteke et al., 1993b). Thus, there is need to determine the level of fertility of Musa

Received for publication 22 Nov. 1995. Accepted for publication 4 June 1996. Paper no. IITA/95/JA/ 36 . The cost of publishing this paper was defrayed in part by the payment of page charges. Under postal regulations, this paper therefore must be hereby marked advertisement solely to indicate this fact. ${ }^{1}$ Research Assistant, Breeder.

${ }^{2}$ Program Leader, Breeder and Geneticist; to whom reprint requests should be addressed. Current address: IITA, c/o Lambourn, 26 Dingwall Rd., Croydon, CR9 3EE, England.

HortSCience, Vol. 31(6), OCTOBER 1996
The screening for apparent male fertility was carried out on 168 Musa accessions: 103 landraces (AA, BB, AAA, AAB, ABB, AAAB) and 65 plantain-banana hybrids. The parents of the hybrids were the AAB plantains 'Bobby Tannap', 'Obino 1' Ewai', and 'Agbagba' French Reversion, and the wild diploid 'Calcutta 4' (Vuylsteke et al., 1993b).

Plants were evaluated in the fields of IITA High Rainfall Station at Onne, southeastern Nigeria. This location has an arable marshy/ swamp vegetation and is in the secondary center of plantain diversification (Vuylsteke et al., 1993a). The station has a well-drained, highly acidic Thionic Fluvisol, which is poor in nutrients. Total annual rainfall averages $2400 \mathrm{~mm}$ and temperature ranges from 25 to $27^{\circ} \mathrm{C}$.

During anthesis, male flowers were excised from the male bud between 7:30 and 10:30 AM (before the heat of the sun began to dry out the sticky pollen) and immediately transported to the laboratory. Pollen grains were manually dislodged from the stamen, spread on a glass slide to generate a thin film, and stained with acetocarmine glycerol jelly. The preparation was then covered with a microscope slip and allowed to stand for $24 \mathrm{~h}$ to allow passive uptake of stain. This slide preparation was then observed under bright field illumination $(\times 400$ magnification $)$ using a Leitz Diaplan binocular microscope.

A total of 200 pollen grains was counted in one to two microscopic field views. Only completely rounded and deeply stained grains were considered viable. The diameters of 10 randomly selected deeply stained pollen grains were measured. Statistical confidence intervals (CI) at $95 \%$ level were calculated for pollen stainability as follows:

$\mathrm{CI}=\left[\mathrm{p}+\left(1.96^{2} / 2 \mathrm{n}\right) \pm 1.96\{[\mathrm{p}(1-\mathrm{p}) / \mathrm{n}]+\right.$ $\left.\left.\left[1.96^{2} / 4 n^{2}\right]\right\}^{1 / 2}\right] /\left\{1+\left[1.96^{2} / \mathrm{n}\right]\right\}$

where $\mathrm{p}$ is the proportion of stained pollen and $\mathrm{n}$ is the total number of pollen grains observed. The quantity of pollen manually dislodged from each stamen was scored on a scale of 0 to 3: $0=$ none $; 1=$ scarce $; 2=$ moderate $3=$ abundant.
Table 1. Average pollen diameter and stainability in $2 x, 3 x$, and $4 x$ Musa germplasm that produce pollen at anthesis.

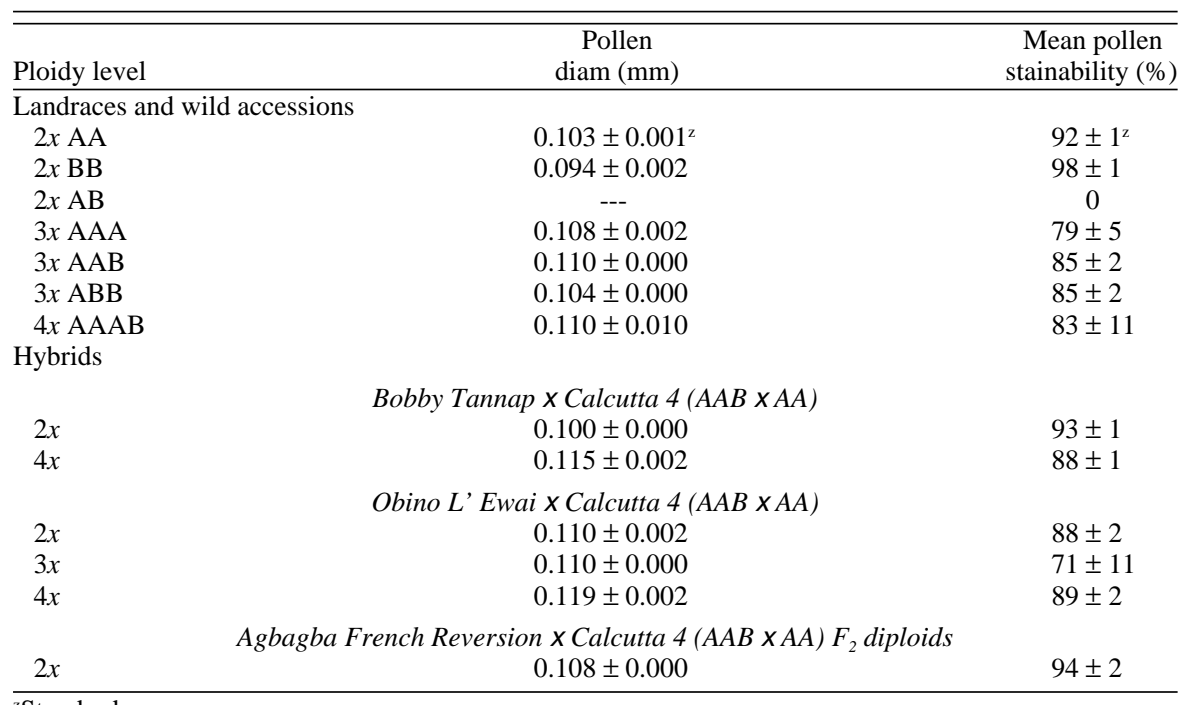

${ }^{2}$ Standard error. 
Simple linear correlation coefficients between pollen quantity and pollen quality were determined to establish the association between these two variables.

\section{Results and Discussion}

Stainability with acetocarmine glycerol is an established method to determine pollen viability for estimating the level of male fertility of Musa clones (Dessauw, 1988). Hence, this technique has been recommended for selection of prospective male parents for further use in Musa breeding schemes.

Among the landraces, the diploids had on average the highest pollen stainability as compared to the triploids and tetraploids (Table 1). These findings suggest that crosses using diploid accessions as male parents are more likely to be successful than crosses with polyploid male parents.

Slight increases in mean pollen diameter due to ploidy level were observed in the landraces and in the hybrids (Table 1). Also, in diploid species, $M$. balbisiana pollen had a larger diameter than $M$. acuminata. This difference agrees with other findings where $M$. acuminata (A genome) had $1.26 \mathrm{pg} /$ nuclear DNA (2C), whereas M. balbisiana (B genome) had 1.14 pg (Afza et al., 1993). This difference may explain why ABB cooking bananas had slightly smaller pollen size than AAB landraces.

Five wild, nonedible AA bananas produced abundant viable pollen, including 'Calcutta 4' and 'Long Tavoy' (Table 2), confirming previous reports (Dodds and Simmonds, 1948; Swennen and Vuylsteke, 1993). Although highly stained pollen (up to $98 \%$ ) occurred in the progenies of male-sterile plantains and 'Calcutta 4', two diploid hybrids lacked pollen (Table 3). This sterility in diploid plantainbanana hybrids results from the interaction of a plantain-sensitive cytoplasm with recessive nuclear genes from banana (Ortiz, 1995).

Lack of pollen production during anthesis was identified in 28 accessions (Tables 2 and $3)$. Half of the male-sterile accessions were diploids. This finding confirms that male sterility in Musa is not exclusive to triploids. At the same time, none of the triploid accessions assessed had high enough levels of pollen production to warrant their use as male stocks in cross breeding (Table 2). Low pollen production in triploids compared to diploids and tetraploids has been attributed to meiotic abnormalities (Ramirez, 1990; Simmonds, 1966). However, this trait also may be under genetic control (Ortiz, 1995) and influenced by the environment (Ulburghs, 1994).

There was a significant correlation between pollen production and stainability in both landraces $(r=0.61, P \leq 0.01)$ and in the hybrids $(r=0.65, P \leq 0.01)$. This significant association in Musa could be physiological and under genetic control, although this correlation may be affected by the environment.

Clones producing at least $25 \%$ viable pollen (completely rounded and deeply stained) at moderate production levels are considered sufficiently male-fertile. This assumption is
Table 2. Assessment in pollen diameter, stainability, and production in Musa landraces and wild accessions.

\begin{tabular}{|c|c|c|c|c|}
\hline Clone & Genome & $\begin{array}{c}\text { Pollen } \\
\text { diam } \\
(\mathrm{mm})\end{array}$ & $\begin{array}{c}\text { Mean pollen } \\
\text { stainability } \\
(\%)\end{array}$ & $\begin{array}{c}\text { Pollen } \\
\text { production }^{2}\end{array}$ \\
\hline \multicolumn{5}{|l|}{ M. acuminata } \\
\hline 'Basilan' & $\mathrm{AA}^{\mathrm{y}, \mathrm{x}}$ & 0.10 & 86 & 1.5 \\
\hline 'Borneo' & AA & 0.10 & 98 & 2.0 \\
\hline 'Calcutta 4' & AA & 0.11 & 100 & 3.0 \\
\hline 'Djum metek' & AA & 0.10 & 96 & 1.5 \\
\hline 'Djum tau' & AA & 0.09 & 89 & 1.5 \\
\hline 'Galeo' & AA & 0.10 & 88 & 2.5 \\
\hline 'Guyod' & AA & 0.11 & 66 & 1.5 \\
\hline Higa III-4 & AA & 0.11 & 94 & 1.5 \\
\hline Higa III-97 & AA & 0.10 & 100 & 2.0 \\
\hline Higa hybrid & AA & 0.10 & 98 & 3.0 \\
\hline Holotype & AA & 0.10 & 100 & 2.0 \\
\hline Hybrid 382 & AA & 0.10 & 98 & 2.0 \\
\hline 'Inarnibal' & AA & 0.12 & 66 & 1.0 \\
\hline 'Long Tavoy' & AA & 0.11 & 100 & 3.0 \\
\hline 'Morong Princesa' & AA & 0.11 & 86 & 1.0 \\
\hline No. 110 & AA & 0.11 & 93 & 1.0 \\
\hline 'Paka' & AA & 0.11 & 92 & 2.5 \\
\hline 'Pa' ('Pathalong') & AA & 0.11 & 96 & 1.5 \\
\hline 'Pa Musore' & AA & 0.10 & 96 & 2.5 \\
\hline 'Pa Songkla' & AA & 0.10 & 100 & 2.5 \\
\hline 'Pisang lilin' & AA & 0.13 & 94 & 2.0 \\
\hline 'Pisang madu' (258) & AA & 0.09 & 97 & 1.5 \\
\hline 'Pisang madu' (276) & AA & 0.10 & 92 & 1.5 \\
\hline 'Pisang madu' (507) & AA & 0.09 & 98 & 2.0 \\
\hline 'Pitu' & AA & 0.11 & 89 & 2.0 \\
\hline 'Selangor' & AA & 0.10 & 97 & 2.5 \\
\hline SF 215 & AA & 0.09 & 86 & 1.0 \\
\hline SF 248 & AA & 0.11 & 88 & 1.5 \\
\hline SF 265 & AA & 0.09 & 66 & 1.0 \\
\hline subspp. banksii (606) & AA & 0.09 & 94 & 2.5 \\
\hline subspp. banksii (619) & AA & 0.10 & 94 & 2.0 \\
\hline subspp. banksii (621) & AA & 0.09 & 98 & 2.0 \\
\hline subspp. banksii (623) & AA & 0.09 & 96 & 2.0 \\
\hline subspp. malaccensis & AA & 0.10 & 98 & 3.0 \\
\hline subspp. malaccensis 'Pahang' & AA & 0.10 & 99 & 2.5 \\
\hline 'Siang Hil' & AA & 0.11 & 88 & 1.0 \\
\hline 'Tjau Lagada' & AA & 0.10 & 93 & 2.0 \\
\hline 'Toowolee' & AA & 0.12 & 88 & 1.5 \\
\hline 'Truncata' (252) & AA & 0.10 & 96 & 2.0 \\
\hline 'Truncata' (393) & AA & 0.10 & 97 & 2.5 \\
\hline 'Tuu Gia' & AA & 0.11 & 75 & 1.0 \\
\hline 'Uwati' & AA & 0.12 & 86 & 1.0 \\
\hline 'Waigu' & AA & 0.10 & 100 & 3.0 \\
\hline 'Who-gu' & AA & 0.12 & 91 & 1.5 \\
\hline \multicolumn{5}{|l|}{ M. balbisiana } \\
\hline 'Butohan 2' & BB & 0.09 & 97 & 2.0 \\
\hline 'Los Banos' & $\mathrm{BB}$ & 0.10 & 99 & 2.5 \\
\hline ‘Tani' & BB & 0.10 & 99 & 2.5 \\
\hline $\mathrm{I}-63$ & $\mathrm{BB}$ & 0.09 & 98 & 2.5 \\
\hline 10852 & $\mathrm{BB}$ & 0.09 & 97 & 2.0 \\
\hline \multicolumn{5}{|l|}{ Dessert banana } \\
\hline 'Biu Ketip' & AAA & 0.10 & 87 & 1.0 \\
\hline 'Green Red' & AAA & 0.12 & 92 & 1.5 \\
\hline 'Highgate' & AAA & 0.12 & 61 & 1.0 \\
\hline 'Jawaka' & AAA & 0.10 & 75 & 1.0 \\
\hline 'Klue Hom Bao' & AAA & 0.11 & 70 & 1.0 \\
\hline 'Lai' & AAA & 0.12 & 90 & 1.5 \\
\hline 'Mambu' & AAA & 0.10 & 74 & 1.0 \\
\hline 'Muga' & AAA & 0.10 & 90 & 1.0 \\
\hline 'Padri' & AAA & 0.10 & 92 & 1.5 \\
\hline 'Pisang masak hijau' & AAA & 0.11 & 30 & 1.0 \\
\hline 'Pisang nangka' & AAA & 0.11 & 86 & 1.5 \\
\hline 'Robusta' & AAA & 0.11 & 88 & 1.0 \\
\hline 'Yangambi Km. 5' & AAA & 0.11 & 89 & 1.5 \\
\hline \multicolumn{5}{|l|}{ Plantains and starchy bananas } \\
\hline 'Dominico Macho' & $\mathrm{AAB}$ & 0.13 & 82 & 1.0 \\
\hline 'Klue roi wee' & $\mathrm{AAB}$ & 0.10 & 82 & 1.0 \\
\hline 'Muracho' & $\mathrm{AAB}$ & 0.10 & 84 & 1.5 \\
\hline 'Pisang Kelat' & $\mathrm{AAB}$ & 0.11 & 91 & 1.5 \\
\hline \multicolumn{5}{|l|}{ Cooking bananas } \\
\hline 'Blue Torres strait 1' & $\mathrm{ABB}$ & 0.10 & 90 & 1.5 \\
\hline
\end{tabular}


Table 2. Continued.

\begin{tabular}{|c|c|c|c|c|}
\hline Clone & Genome & $\begin{array}{c}\text { Pollen } \\
\text { diam } \\
(\mathrm{mm})\end{array}$ & $\begin{array}{c}\text { Mean pollen } \\
\text { stainability } \\
(\%)\end{array}$ & $\begin{array}{c}\text { Pollen } \\
\text { production }\end{array}$ \\
\hline 'Bluggoe' & $\mathrm{ABB}$ & 0.10 & 68 & 1.0 \\
\hline 'Espermo' & $\mathrm{ABB}$ & 0.11 & 66 & 1.0 \\
\hline 'Pisang Awak' & $\mathrm{ABB}$ & 0.12 & 80 & 1.0 \\
\hline 'Maduranga' & $\mathrm{ABB}$ & 0.10 & 88 & 1.0 \\
\hline 'Mothan/Saba' & $\mathrm{ABB}$ & 0.10 & 84 & 1.0 \\
\hline 'Sabra' & $\mathrm{ABB}$ & 0.10 & 87 & 1.0 \\
\hline 'Simili Radjah' & $\mathrm{ABB}$ & 0.10 & 87 & 1.0 \\
\hline \multicolumn{5}{|l|}{ Tetraploid landraces } \\
\hline 'Ngern' & AAAB & 0.12 & 94 & 1.0 \\
\hline 'Ouro da mata' & $\mathrm{AAAB}$ & 0.10 & 72 & 1.0 \\
\hline \multicolumn{5}{|l|}{ Others } \\
\hline Unknown 'Mainz' & $?$ & 0.10 & 92 & 1.5 \\
\hline
\end{tabular}

${ }^{2} 0=$ none; 1 = scarce; 2 = moderate; 3 = abundant.

'Without pollen at anthesis: AA: A3366-5, 'Bandjim', 'Figue Sucree', 'Gu-nin-chio', 'Pisang Berlin', 'Pisang mas', 'Pisang mas ayer', 'Putewey', 'Racadag', 'Thong Det'; AB: 'Kamaramasenge', 'Kisubi'; AAA: 'Palang'; AAB: 'Corne Plantain'/'Maia Maoli', 'Mbeta1/Mysore', 'Pisang raja', 'Pome', 'Popoulou', 'Rajapuri India'; ABB: 'Cardaba', 'Fougamou', 'Foulah 4', 'Ice cream', 'Nzizi', 'Pelipita', 'Pelipita Majoncho'.

${ }^{\mathrm{x}}$ Musa acuminata and M. balbisiana are donors of the A and B genomes, respectively.

Table 3. Assessment in pollen diameter, stainability, and production in plantain-derived hybrids.

\begin{tabular}{|c|c|c|c|c|}
\hline Clone & Ploidy & $\begin{array}{c}\text { Pollen } \\
\text { diam } \\
(\mathrm{mm})\end{array}$ & $\begin{array}{c}\text { Mean pollen } \\
\text { stainability } \\
(\%)\end{array}$ & $\begin{array}{c}\text { Pollen } \\
\text { production }\end{array}$ \\
\hline \multicolumn{5}{|c|}{ Agbagba French Reversion $\times$ Calcutta $4(A A B \times A A) F_{2}$ diploids } \\
\hline $8256-4$ & $2 x$ & 0.11 & 98 & 2.5 \\
\hline $8256-8$ & $2 x$ & 0.10 & 78 & 2.0 \\
\hline $8096-12$ & $2 x$ & 0.11 & 96 & 1.5 \\
\hline $8096-13$ & $2 x$ & 0.11 & 95 & 1.0 \\
\hline $8096-22$ & $2 x$ & 0.11 & 92 & 1.0 \\
\hline $8096-26$ & $2 x$ & 0.11 & 98 & 2.0 \\
\hline \multicolumn{5}{|c|}{ Bobby Tannap $\times$ Calcutta $4(A A B \times A A)$} \\
\hline K46-1 & $2 x$ & 0.12 & 81 & 1.5 \\
\hline $1135-1$ & $2 x$ & 0.11 & 91 & 1.5 \\
\hline $1487-3$ & $2 x$ & 0.11 & 82 & 1.0 \\
\hline $1489-3$ & $2 x$ & 0.11 & 98 & 2.0 \\
\hline $1518-4$ & $2 x$ & 0.11 & 98 & 2.0 \\
\hline $1657-12$ & $2 x$ & --- & 0 & 0.0 \\
\hline $1659-2$ & $2 x$ & 0.10 & 96 & 2.0 \\
\hline $1668-7$ & $2 x$ & 0.11 & 96 & 2.0 \\
\hline $2085-1$ & $2 x$ & 0.11 & 94 & 2.0 \\
\hline $2625-5$ & $2 x$ & 0.10 & 80 & 2.5 \\
\hline $2625-20$ & $2 x$ & 0.10 & 96 & 2.5 \\
\hline $2829-62$ & $2 x$ & 0.12 & 90 & 1.5 \\
\hline $3564-7$ & $2 x$ & 0.11 & 94 & 2.0 \\
\hline $3564-8$ & $2 x$ & 0.11 & 88 & 1.5 \\
\hline $4250-1$ & $2 x$ & 0.11 & 90 & 1.0 \\
\hline $4250-2$ & $2 x$ & 0.10 & 94 & 1.5 \\
\hline $4281-2$ & $2 x$ & 0.11 & 96 & 2.0 \\
\hline $4281-3$ & $2 x$ & 0.13 & 96 & 2.0 \\
\hline $4400-3$ & $2 x$ & 0.11 & 99 & 2.5 \\
\hline $4400-5$ & $2 x$ & 0.11 & 96 & 2.0 \\
\hline $4400-7$ & $2 x$ & 0.13 & 92 & 1.0 \\
\hline $4400-8$ & $2 x$ & 0.12 & 98 & 2.5 \\
\hline $4600-12$ & $2 x$ & 0.12 & 92 & 1.5 \\
\hline $4600-15$ & $2 x$ & 0.11 & 94 & 1.5 \\
\hline 4800-1 & $2 x$ & 0.12 & 95 & 1.5 \\
\hline $4885-1$ & $2 x$ & 0.12 & 90 & 2.0 \\
\hline $5233-1$ & $2 x$ & 0.11 & 92 & 2.0 \\
\hline $5233-2$ & $2 x$ & 0.10 & 88 & 1.5 \\
\hline $5837-1$ & $2 x$ & 0.11 & 100 & 2.5 \\
\hline $9007-3$ & $2 x$ & --- & 0 & 0.0 \\
\hline $9007-4$ & $2 x$ & 0.13 & 98 & 2.0 \\
\hline $10033-5$ & $2 x$ & 0.10 & 98 & 2.0 \\
\hline $1187-8$ & $3 x$ & 0.10 & 88 & 1.0 \\
\hline $582-4$ & $4 x$ & 0.12 & 82 & 1.0 \\
\hline $2796-5$ & $4 x$ & 0.11 & 91 & 1.5 \\
\hline $2870-20$ & $4 x$ & 0.12 & 91 & 2.0 \\
\hline $4479-1$ & $4 x$ & 0.11 & 88 & 2.5 \\
\hline $7261-3$ & $4 x$ & 0.11 & 92 & 2.5 \\
\hline $8223-1$ & $4 x$ & 0.12 & 91 & 1.5 \\
\hline
\end{tabular}

continued on nextpage based on the observation that 'Maraw', an edible banana with this level of male fertility (UIburghs, 1994), was a functional male parent that produce true viable seeds when its pollen was used in crosses with 'Mbi Egome', a female-fertile triploid plantain (Vuylsteke et al., 1993b). Conversely, the black sigatokaresistant cultivar Tuu Gia had scarce pollen production, with $75 \%$ viable, and has not yielded any seed despite its consecutive crossing to several female-fertile partners over 1 year (data not shown). Other factors, in addition to pollen fertility, probably influence seedset and viability (Ortiz and Vuylsteke, 1995). It seems that, in some Musa accessions, quantity of viable pollen is very important to ensure seed production. Further investigation may establish if the quantity and quality of pollen produced by male parents affect seedset in hand pollinations with female-fertile partners across sites and seasons.

\section{Literature Cited}

Afza, R., D. Kammer, J. Dolezel, J. Konig, M. van Duren, G. Kahl, and F. Novak. 1993. The potential of nuclear DNA flow-cytometry and DNA fingerprinting for Musa improvement programmes, p. 65-75. In: J. Ganry (ed.). Breeding banana and plantain for resistance to diseases and pests. CIRAD in collaboration with INIBAP, Montpellier, France.

De Langhe, E. 1987. Towards an international strategy for genetic improvement in the genus Musa, p. 19-23 In: G.J. Persley and E. De Langhe (eds.). Banana and plantain breeding strategies. Proc. Intl. Wkshp., Cairns, Australia, 13-17 Oct. 1986. Proc. 21 ACIAR, Canberra, Australia.

Dessauw, D. 1988. Etude des facteurs de la sterilite du bananier (Musa spp.) et des relations citotaxonomiques entre $M$. acuminata et $M$. balbisiana Colla. Fruits 43:539-558, 615-638, 685-700.

Dodds, K.S. and N.W. Simmonds. 1948. Sterility and parthenocarpy in diploid hybrids of Musa. Heredity 2:121-117.

Mobambo, K.N., F. Gauhl, D. Vuylsteke, R. Ortiz, C. Pasberg-Gauhl, and R. Swennen. 1993. Yield loss in plantain from black sigatoka leaf spot and field performance of resistant hybrids. Field Crops Res. 35:35-42.

Mukasa, S.B. and P.R. Rubahaiyo. 1993. Male fertility in Uganda banana germplasm. African Crop Sci. J. 1:87-93.

Ortiz, R. 1995. Musa genetics, p. 84-109. In: S. Gowen (ed.). Bananas and plantains. Chapman \& Hall, London.

Ortiz, R. and D. Vuylsteke. 1995. Factors influencing seed set in triploid Musa spp. L. and production of euploid hybrids. Ann. Bot. 75:151155.

Plantain and Banana Improvement Program. 1993. 1992 Annual report of the Plantain and Banana Improvement Program. Crop Improvement Div., Intl. Inst. Trop. Agr., Ibadan, Nigeria.

Ramirez, D.A. 1990. A cytological survey of Philippine bananas, p. 92-107. In: R.L. Jarret (ed.). Identification of genetic diversity in the genus Musa. Proc. Intl. Wkshp., Los Banos, Philippines, 5-10 Sept. 1988. INIBAP, Montpellier, France.

Sathiamoorthy, S. 1994. Musa improvement in India, p. 188-200. In: D. Jones (ed.). The improvement and testing of Musa: A global partnership. Proc. First Global Conf. Intl. Musa Testing 
Breeding, Cultivars, Rootstocks, \& Germplasm Resources

Table 3. Continued.

\begin{tabular}{|c|c|c|c|c|}
\hline Clone & Ploidy & $\begin{array}{c}\text { Pollen } \\
\text { diam } \\
(\mathrm{mm})\end{array}$ & $\begin{array}{c}\text { Mean pollen } \\
\text { stainability } \\
(\%)\end{array}$ & $\begin{array}{c}\text { Pollen } \\
\text { production }\end{array}$ \\
\hline \multicolumn{5}{|c|}{ Obino l'Ewai $\times$ Calcutta $4(A A B \times A A)$} \\
\hline $565-16$ & $2 x$ & 0.11 & 88 & 1.0 \\
\hline $566-32$ & $2 x$ & 0.10 & 80 & 1.5 \\
\hline $1199-6$ & $2 x$ & 0.11 & 88 & 2.5 \\
\hline 1448-1 & $2 x$ & 0.12 & 91 & 1.5 \\
\hline $1549-5$ & $2 x$ & 0.11 & 86 & 1.5 \\
\hline $1549-7$ & $2 x$ & 0.10 & 85 & 1.5 \\
\hline $1586-2$ & $2 x$ & 0.11 & 91 & 1.5 \\
\hline $5620-1$ & $2 x$ & 0.12 & 96 & 1.5 \\
\hline $9243-2$ & $2 x$ & 0.12 & 62 & 1.0 \\
\hline $9243-5$ & $2 x$ & 0.10 & 94 & 2.0 \\
\hline $14558-1$ & $2 x$ & 0.11 & 98 & 2.0 \\
\hline $1199-1$ & $3 x$ & 0.11 & 82 & 1.0 \\
\hline $14676-2$ & $3 x$ & 0.11 & 60 & 1.0 \\
\hline $597-4$ & $4 x$ & 0.13 & 78 & 1.0 \\
\hline $1621-1$ & $4 x$ & 0.11 & 91 & 2.0 \\
\hline $2637-49$ & $4 x$ & 0.12 & 83 & 1.5 \\
\hline 4424-4 & $4 x$ & 0.11 & 85 & 1.0 \\
\hline $4698-1$ & $4 x$ & 0.12 & 94 & 2.0 \\
\hline 4744-1 & $4 x$ & 0.12 & 92 & 2.0 \\
\hline 5706-1 & $4 x$ & 0.12 & 88 & 2.0 \\
\hline $5860-1$ & $4 x$ & 0.12 & 91 & 2.0 \\
\hline $6562-1$ & $4 x$ & 0.12 & 95 & 2.0 \\
\hline $7002-1$ & $4 x$ & 0.12 & 90 & 2.0 \\
\hline
\end{tabular}

${ }^{\mathrm{z}} 0=$ none; 1 = scarce $; 2$ = moderate 3 = abundant.
Program. FHIA, Honduras, 27-30 Apr. 1994. INIBAP, Montpellier, France.

Simmonds, N.W. 1966. Bananas tropical agriculture series. 2nd ed. Longmans, London and New York.

Stover, R.H. 1983. Effet du Cercospora noir sur les plantains en Amerique Centrale. Fruits 38:326329.

Swennen, R. and D. Vuylsteke. 1993. Breeding black sigatoka resistant plantains with a wild banana. Tropical Agr. 70:74-77.

Ulburghs, F. 1994. Inleidende studie van de pollenvariabiliteit in Musa. Ir. MS Thesis, Catholic Univ. of Leuven (KUL), Belgium.

Vuylsteke, D., R. Ortiz, and S. Ferris. 1993a. Genetic and agronomic improvement for sustainable production of plantain and banana in subSaharan Africa. African Crop Sci. J. 1:1-8.

Vuylsteke, D., R. Swennen, and R. Ortiz.1993b. Development and performance of black sigatokaresistant tetraploid hybrids of plantains ( $M u s a$ spp. AAB group). Euphytica 65:33-42. 